\title{
Clinical Use of a Mutual Information-Based Automated Image Registration System for Conformal Radiotherapy Treatment Planning
}

\author{
Marc Kessler, $\mathrm{PhD}^{1}$, Janelle Solock ${ }^{1}$, Paul Archer ${ }^{1}$, and Charles Meyer, $\mathrm{PhD}^{2}$ \\ Department of ${ }^{1}$ Radiation Oncology and ${ }^{2}$ Radiology \\ The University of Michigan Medical School, Ann Arbor, Michigan, USA 48109 \\ \{MKessler, Jsolack, PGArcher, CMeyer\}@umich.edu
}

\begin{abstract}
In this paper we describe the clinical use of an in-house mutual information-based image registration system for improving target volume definition in conformal radiotherapy treatment planning. This system enables a clinician to delineate clinical and anatomic structures on magnetic resonance and nuclear medicine imaging studies and have these structures geometrically mapped to the $\mathrm{x}$-ray CT study used for treatment planning. This system requires very little user interaction and can accommodate a wide range of anatomic sites. These factors combined with consistent accuracy at the sub-voxel to voxel level have improved the overall quality and physician confidence in the delineation of target volumes and surrounding healthy tissues.
\end{abstract}

\section{Introduction}

In conformal radiation therapy, accurate delineation of the target volume and surrounding healthy tissues is essential. Modern treatment machines are now capable of delivering highly shaped doses of radiation to a prescribed region while minimizing doses to adjacent tissues. Although x-ray CT is still the primary modality for planning these radiation treatments, other modalities such as magnetic resonance imaging and nuclear medicine studies can provide important data for improving target volume localization. While the practice of incorporating information from multiple modalities into the treatment planning process is by no means new and while most commercial treatment planning systems now offer tools to support this, routine clinical use of these tools is still limited to a few anatomic sites and imaging situations. Requirements placed on the input image data extent, amount of preprocessing involved and the lack of appropriate geometric transforms have confounded broader use of these tools. In order to exploit a wider range of anatomic and functional imaging studies for radiotherapy treatment planning, we have implemented a mutual information-based image registration system. This system requires very little user interaction, is robust to limited and missing data and supports both affine and thin-plate spline geometric transformations [1]. The details of this system and the underlying algorithms are reported elsewhere [2]. In this paper we demonstrate the use of this system using two clinical examples. 


\section{Clinical Examples}

Two clinical cases are described to illustrate the use of the registration system for radiotherapy treatment planning. One case involves a benign menengioma that enhances well on MR but is difficult to delineate on the treatment planning CT. The other case involves the use of functional image data from PET to help resolve uncertainties in a CT-based lung tumor target volume.

\subsection{MR - CT Case}

This case involves the registration of a diagnostic MR with a planning CT for a patient with a benign meningioma located near the base of the skull (Fig. 1). As is often the case, the orientation of the patient's head in the two imaging studies was different. As is often the case, the patient was imaged wearing a custom-made immobilization mask during the CT procedure but not during the MR because of the space constraints of the head coil. Although the MR data adequately covered the involved region it did not include the entire cranium. While this situation might have limited the accuracy of a surface-based registration, the MI-based registration behaved properly and was judged to be accurate to within one pixel $(1 \mathrm{~mm})$. In addition to the value of the both the axial and coronal MR data for delineating the target volume, the coronal MR was essential for accurate localization of the optic nerves and chiasm.

\subsection{PET - CT Case}

This case involves a large tumor in the upper portion of the right lung (Fig. 2). Using only the CT data, the clinician defined the target volume. The clinician expressed uncertainty about the detailed extent of parts of the tumor, especially in regions containing CT streak artifacts caused by nearby bones. PET data was acquired to help resolve these uncertainties. A PET-only target volume was delineated and mapped to the CT dataset using a transformation computed using mutual information. A comparison of these volumes is shown in Figure 2. The PET defined target volume not only helped to clarify the superior shape of the tumor (Fig 2-B) but also revealed a portion of the tumor missed in the CT-only target volume (Fig 2-A).

\section{Summary}

A mutual information-based automatic multimodality image registration system has implemented and applied across a broad spectrum of volumetric data sets of various modalities and anatomies. A practical advantage of this system is the minimal user interaction and data pre-processing required. The major strength, however, is the robustness of the mutual information-based registration metric to sparse or missing data and the ability to register anatomic data with functional data where the precise locations of anatomic features are often difficult to define. These benefits, combined with option of using geometric warping in addition to affine transformations to model certain tissue deformation and machine distortions greatly expands the range of imaging studies that the clinical can quantitatively use to improve the accuracy of target volume and normal tissue delineation for radiotherapy treatment planning. 

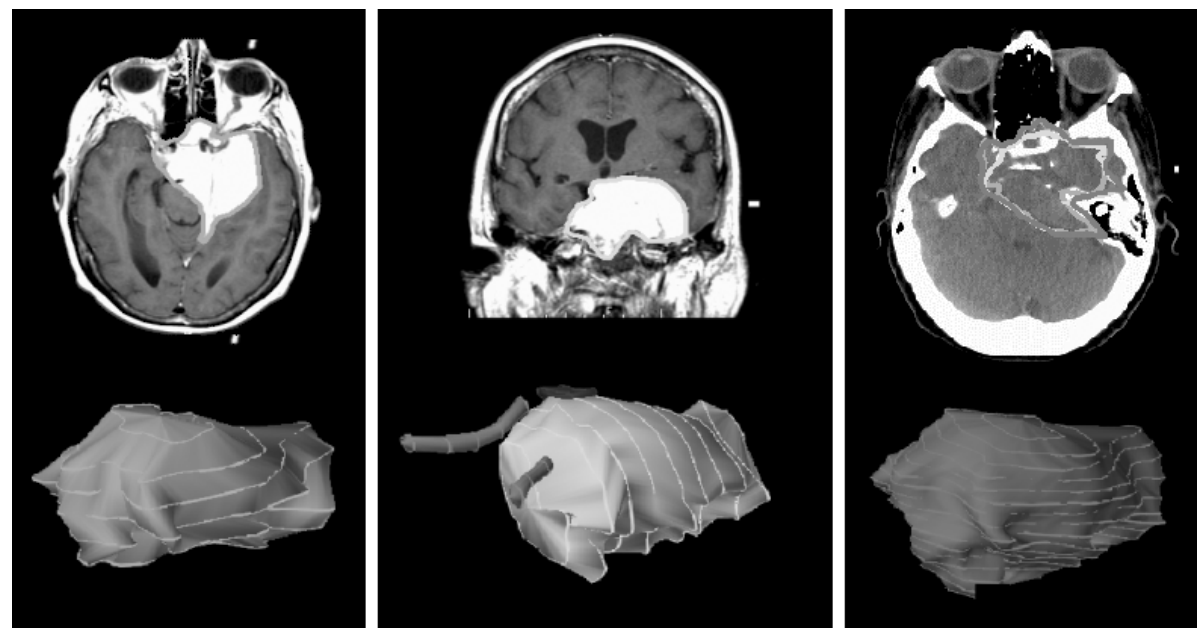

Fig 1. Delineation of target volume and normal tissues using axial (left) and coronal (center) MR imaging series. For this case, the CT target volume (right) was constructed from the logical combination of the mapped structures from the coronal and axial MR data.
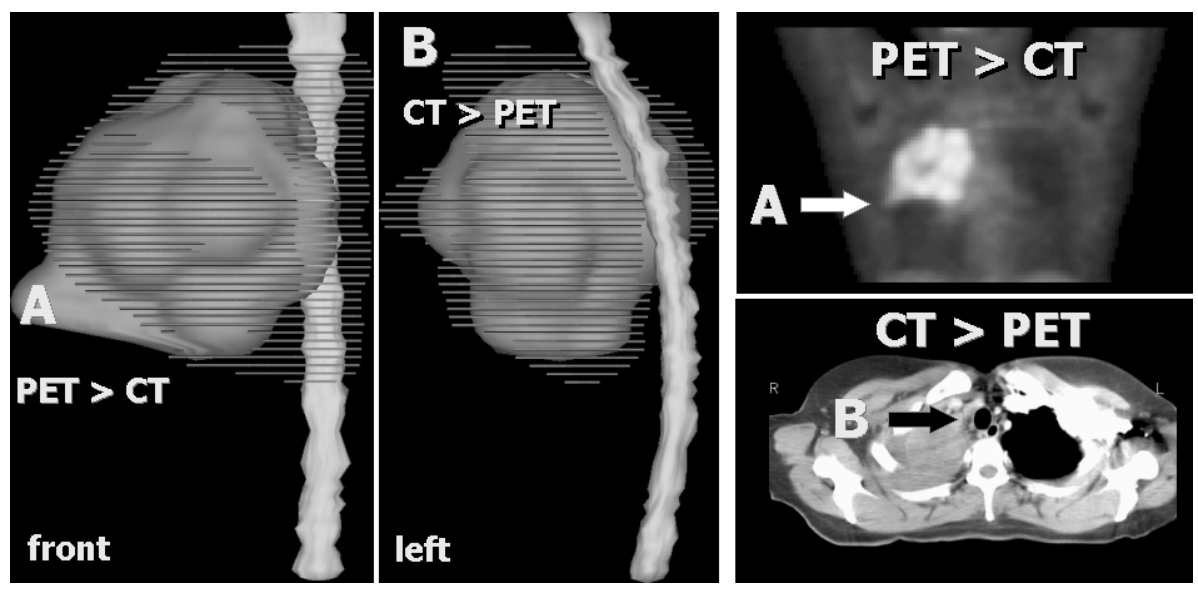

Fig 2. Target volume definition from CT only (contours) and PET only (surface). The long vertical structure is the CT-based spinal cord.

\section{References}

1. Bookstein F L 1991. Morphometric tools for landmark data: geometry and biology. Cambridge Univ. Press: New York. 435 pp.

2. Meyer C R, Boes JL, Kim B, Bland B H, et al. 1997, Demonstration of accuracy and clinical versatility of mutual information for automatic multimodality image fusion using affine and thin plate spline warped geometric deformations. Medical Image Analysis 1(3):195-206. 